\title{
ON THE PARABOLIC POTENTIALS IN DEGENERATE-TYPE HEAT EQUATION ${ }^{1}$
}

\author{
IGOR MALYSHEV \\ Department of Mathematics and Computer Science \\ San Jose State University \\ San Jose, CA 95192
}

\begin{abstract}
Using distributions theory technique we introduce parabolic potentials for the heat equation with one time-dependent coefficient (not everywhere positive and continuous) at the highest space-derivative, discuss their properties, and apply obtained results to three illustrative problems. Presented technique allows to deal with some equation of the degenerate/mixed type.
\end{abstract}

Key words: parabolic potentials, variable coefficient, boundary value problems, equations of degenerate/mixed type.

AMS subject classifications: 35K65, 35R05.

\section{INTRODUCTION}

In this paper we shall study the properties of "parabolic" potentials associated with the boundary value problems in a semi-infinite domain of the following type:

$$
\mathrm{L}_{\alpha} \mathrm{u}=\frac{\partial \mathrm{u}}{\partial \mathrm{t}}-\alpha(\mathrm{t}) \frac{\partial^{2} \mathrm{u}}{\partial \mathrm{x}^{2}}=\mathrm{f}(\mathrm{x}, \mathrm{t}), \mathrm{x}>0, \mathrm{t}>0
$$

$$
\mathrm{u}(\mathrm{x}, 0)=\varphi(\mathrm{x}), \mathrm{x} \geq 0
$$

$$
\mathrm{u}(0, \mathrm{t})=\mathrm{r}(\mathrm{t}), \mathrm{t} \geq 0 ; \quad(\varphi(0)=\mathrm{r}(0))
$$

\footnotetext{
${ }^{1}$ Received: August 1990, Revised: February 1991
} 
Throughout the paper the coefficient $\alpha(t) \in L_{1}[0, T]$, is not necessarily positive (which implies that (1) may be of degenerate/mixed type), is defined everywhere in $[0, T]$ and satisfies one of the following conditions:

(i) $\alpha(t) \geq 0$, with equality allowed only at isolated points that do not cluster anywhere in $[0, T]$;

(ii) $\alpha_{1}(t)$ defined by the formula

$$
\alpha_{1}(t)=\int_{0}^{t} \alpha(z) d z, \quad\left(\alpha_{1}(0)=0\right)
$$

is positive for all $t>0$, which allows $\alpha(t)$ to be even negative in some intervals.

Obviously, any function satisfying (i) is a function of the (ii) type.

It should be noted that in neither case (for different reasons) (1) is reducible to a standard heat operator $u_{t}-u_{x x}$. The realization of this comes from the relatively obvious substitution of variables [1]:

$$
\tau=\int_{0}^{t} \alpha(z) d z
$$

which in case of (i) implies existence of inverse function $t(\tau)$ with a finite derivative $t_{\tau}^{\prime}$ $=1 / \alpha(t)$ at the points where $\alpha(t) \neq 0$. In (ii) case inversion is not possible at all. To get around this obstacle, we derive the fundamental solution, potentials and their properties, and solution of (1)-(3) directly from (1) in its original form.

The boundary $S$ of the domain consists of two parts denoted throughout by $S_{1}=$ $\{x \geq 0, t=0\}$ and $S_{2}=\{x=0, t \geq 0\}$. And, finally, $M$ denotes the class of bounded in any strip $(-\infty<\mathrm{x}<\infty) \times[0, \mathrm{~T}]$ functions, vanishing at $\mathrm{t}<0$.

Under condition (ii) the fundamental solution of (1) can be found by applying Fourier transform in $\mathrm{x}$ in the form [1]:

(4) $\quad E_{\alpha}(x, t)=E\left(x, \alpha_{1}(t)\right)=\frac{H(t)}{2 \sqrt{\pi \alpha_{1}(t)}} \exp \left(-x^{2} / 4 \alpha_{1}(t)\right)$, 
(were $H(t)$ is Heaviside function), provided that $\alpha_{1}(t)>0$.

Function (4) has the properties similar to those of standard fundamental solution of heat operator [2], such as

$$
\int_{-\infty}^{+\infty} E_{\alpha}(x, t) d x=1 ; E_{\alpha}(x, t) \rightarrow \delta(x) \text { with } t \rightarrow 0^{+}
$$

Denoting $f$, $u$, etc. the functions in (1)-(3) extended as $\equiv 0$ for $x<0, t<0$, the initial-boundary value problem can be put into generalized form

$$
\begin{aligned}
L_{\alpha} \tilde{u} & =\tilde{f}(x, t)+[\bar{u}]_{s_{1}} \cos \left(\bar{n}, \bar{e}_{1}\right) \delta_{s_{1}}-\alpha(t)\left[\frac{\partial \bar{u}}{\partial x}\right]_{s_{2}} \cos \left(\bar{n}, \bar{e}_{2}\right) \delta_{s_{2}} \\
& -\frac{\partial}{\partial x}\left(\alpha(t)[\tilde{u}]_{s_{2}} \cos \left(\bar{n}, \bar{e}_{2}\right) \delta_{s_{2}}\right) \equiv F(x, t)
\end{aligned}
$$

where $[u]_{S}$ is a jump of $u$ on $S=S_{1} \cup S_{2}, n$ is an external normal to $S, e_{1}, e_{2}$ are unit vectors along $t, x$-axis respectively and distributions in the form $\mu \delta_{s},-\left(\mu \delta_{s}\right)_{x}^{\prime}$ are single and double layers in terms of [2].

Since the operator $\mathrm{L}_{\alpha}$ contains a non-constant coefficient, it is not immediately clear whether solution of (6) can be found in the form $u=E_{\alpha} * F$, as in the case of a constant coefficient. However, we still have the following

LEMMA . Under the condition (i) the distributional solution of (6) is unique and can be represented as a convolution of the fundamental solution $E_{\beta}$ ("dual" to $E_{\alpha}$ ) with the right-hand side of (6), that is $\quad u=E_{\beta} * F, \quad$ where, as in [1],

$$
E_{\beta}(x-\xi, t-\tau)=E\left(x-\xi, \beta_{1}(t-\tau)\right),
$$

and

$$
\beta_{1}(t-\tau)=\int_{\tau}^{t} \alpha(z) d z=\alpha_{1}(t)-\alpha_{1}(\tau) ; \beta_{1}(t)=\alpha_{1}(t)
$$


In other words, we treat $\alpha_{1}(t)$ as if it were time variable in a standard case. Obviously, $\beta_{1}$ is continuous and, due to (i) $\beta_{1}(t-\tau)>0$ for $t-\tau>0$.

Proof. Let condition (i) hold. Then $\mathrm{E}_{\beta}(\mathrm{x}-\xi, \mathrm{t}-\tau)$ from (7) is a distributional solution of

$$
L_{\alpha} E_{\beta}(x-\xi, t-\tau)=\frac{\partial E}{\partial t}-\alpha(t) \frac{\partial^{2} E}{\partial x^{2}}=\delta(x-\xi, t-\tau) \text { in } x, t,
$$

and

$$
L_{\alpha}^{+} E_{\beta}(x-\xi, t-\tau)=-\frac{\partial E}{\partial \tau}-\alpha(\tau) \frac{\partial^{2} E}{\partial \xi^{2}}=\delta(x-\xi, t-\tau) \text { in } \xi, \tau .
$$

Verification can be easily done by the Fourier transform technique. Then, using integration by parts we find that $\mathrm{u}=\mathrm{E}_{\beta} * \mathrm{~L}_{\alpha} \mathrm{u}$, and by the direct differentiation $\mathrm{u}=$ $\mathrm{L}_{\alpha}\left(\mathrm{E}_{\beta} * \mathrm{u}\right)$, which leads to:

$$
L_{\alpha}\left(E_{\beta} * \tilde{u}\right)=\left(L_{\alpha} E_{\beta}\right) * \tilde{u}=E_{\beta} * L_{\alpha} \tilde{u}
$$

and the uniqueness of the distributional solution follows immediately, since

$$
\mathrm{L}_{\alpha} \mathrm{u}=0 \Rightarrow \mathrm{E}_{\beta} * \mathrm{~L}_{\alpha} \mathrm{u}=\mathrm{L}_{\alpha} \mathrm{E}_{\beta} * \mathrm{u}=\delta * \mathrm{u}=\mathrm{u}=0
$$

Later we also find that in case of $\mathrm{f}$ and $\mathrm{r}$ in (1)-(3) being zero, the condition (i) here can be relaxed into (ii).

As a result of Lemma, we obtain the following integral representation for the solution of (6) $(x>0, t>0)$ :

$$
\begin{gathered}
u(x, t)=\int_{0}^{t} d \tau \int_{0}^{\infty} f(\xi, \tau) E_{\beta}(x-\xi, t-\tau) d \xi+\int_{0}^{\infty} u(\xi, 0) E_{\beta}(x-\xi, t) d \xi \\
+\left.\int_{0}^{t} \alpha(\tau) u(0, \tau) \frac{\partial}{\partial \xi}\left(E_{\beta}(x-\xi, t-\tau)\right)\right|_{\xi=0} d \tau-\int_{0}^{t} \alpha(\tau) \frac{\partial u}{\partial \xi}(0, \tau) E_{\beta}(x, t-\tau) d \tau .
\end{gathered}
$$


Formula (8) (see also [2]) motivates the following definition of parabolic potentials associated with the boundary value problem (1)-(3):

a) volume potential

(9) $\quad V(x, t)=E_{\beta} * \tilde{f}=\int_{0}^{t} d \tau \int_{0}^{\infty} f(\xi, \tau) E_{\beta}(x-\xi, t-\tau) d \xi$;

b) single-layer potential concentrated on $S_{1}=\{x \geq 0, t=0\}$

$$
V^{(0)}(x, t)=E_{\beta} *\left(\bar{\varphi} \delta_{s_{1}}\right)=\int_{0}^{\infty} \varphi(\xi) E_{\beta}(x-\xi, t) d \xi
$$

c) single-layer potential concentrated on $S_{2}=\{x=0, t \geq 0\}$

$$
V^{(1)}(x, t)=E_{\beta} *\left(\alpha \mu \delta_{s_{2}}\right)=\int_{0}^{t} \alpha(\tau) \mu(\tau) E_{\beta}(x, t-\tau) d \tau
$$

d) double-layer potential concentrated on $S_{2}$

$$
W(x, t)=-\frac{\partial}{\partial x}\left(\alpha r \delta_{s_{2}}\right) * E_{\beta}=\left.\int_{0}^{t} \alpha(\tau) r(\tau) \frac{\partial}{\partial \xi}\left(E_{\beta}(x-\xi, t-\tau)\right)\right|_{\xi=0} d \tau
$$

\section{VOLUME POTENTIAL}

Volume potential $V(x, t)$ given by (9) - is a part of a boundary value problem solution that corresponds to the source-function $f(x, t)$.

THEOREM 1. Let $\alpha(t) \in L_{1}[0, T]$ and satisfy condition (i). Then: (a) for $f \in$ $M, V(x, t) \in M$; (b) for $x \geq 0, t \geq 0 \quad V(x, t)$ is a distributional solution of (1), satisfying zero initial condition as $t \rightarrow 0^{+}$; (c) if extension $f \in C^{2}$ for all $x$ and $t \geq 0$ (which in particular implies that $\left.f(0, t)=f_{x}(0, t)=0\right)$ and all its derivatives up to the second order belong to $M$, then $V_{x x}(x, t)$ is continuous in $\{x \geq 0, t \geq 0\}, V_{t}$ exists for all $x$ and $t$, is continuous in $x$, and its smoothness in $t$ is determined by that of $\alpha(t)$ itself; thus, if in addition $\alpha(t) \in C\left(R_{+}\right)$, then $V(x, t)$ satisfies (1) in the classical sense. 
Proof. Introducing in (9) a new variable y $\left(\beta_{1}(t-\tau)>0\right.$ for $\left.t-\tau>0\right)$

$$
x-\xi=2 \text { y } \sqrt{\beta_{1}(t-\tau)},
$$

for $x \geq 0, t \geq 0$ we express $V(x, t)$ in the form

$$
V(x, t)=\frac{1}{\sqrt{\pi}} \int_{0}^{t} d \tau \int_{-\infty}^{\frac{x}{2 \sqrt{\beta_{1}(t-\tau)}}} f\left(x-2 y \sqrt{\beta_{1}(t-\tau)} ; \tau\right) e^{-y^{2}} d y
$$

and its time-derivative $(t>0)$ :

$$
\begin{aligned}
& \frac{\partial V}{\partial t}=f(x, t) \\
& -\frac{\alpha(t)}{\sqrt{\pi}} \int_{0}^{t} d \tau \int_{-\infty}^{\frac{x}{2 \sqrt{\beta_{1}(t-\tau)}}} f^{\prime} \text { arg.1 }\left(x-2 y \sqrt{\beta_{1}(t-\tau)} ; \tau\right) \frac{y}{\sqrt{\beta_{1}(t-\tau)}} e^{-y^{2}} d y .
\end{aligned}
$$

Using properties of integrals with parameters, it follows from (13)-(14) that $\mathrm{V}(\mathrm{x}, \mathrm{t}) \in$ $C^{2}(x \geq 0, t>0) \cap C^{1}(x \geq 0, t \geq 0)$ for $f$ and $\alpha$ satisfying conditions (c). At the same time $\mathrm{V}$, being a distributional solution of $\mathrm{L}_{\alpha} \mathrm{V}=\mathrm{f}$ and sufficiently smooth, is its classical solution (Du Bois Reimond theorem).

Then, since $f \in M$ and $E_{\beta}$ (as $E_{\alpha}$ ) satisfies (5),

$$
|V(x, t)| \leq\|f\| \int_{0}^{t} d \tau \int_{-\infty}^{+\infty} E_{\beta} d \xi \leq t\|f\|
$$

It follows immediately that $\mathrm{V} \in \mathrm{M}$ and satisfies zero initial condition. The rest of (b) can be obtained as in Lemma, since

$$
\tilde{\mathrm{f}}=\delta * \overline{\mathrm{f}}=\mathrm{L}_{\alpha} \mathrm{E}_{\beta} * \overline{\mathrm{f}}=\mathrm{L}_{\alpha}\left(\mathrm{E}_{\beta} * \tilde{\mathrm{f}}\right)=\mathrm{L}_{\alpha} \mathrm{V}
$$




\section{SINGLE-LAYER POTENTIALS}

(A) Single-layer potential $V^{(0)}(x, t)$, given by (10), is a part of a solution corresponding to the initial condition (2).

THEOREM 2. Let now the condition (ii) hold. Then: (a) for $\varphi \in M, V^{(0)} \in \mathrm{M}$;

(b) $\mathrm{V}^{(0)}$ is a distributional solution of the equation $\mathrm{L}_{\alpha} \mathrm{u}=\varphi \delta_{\mathrm{S} 1}$ and satisfies the initial condition $\mathrm{V}^{(0)}(\mathrm{x}, \mathrm{t}) \rightarrow \varphi(\mathrm{x})$ as $\mathrm{t} \rightarrow 0^{+}$for $\mathrm{x}>0$; (c) if extension $\varphi \in \mathrm{C}^{2}$ (which implies that $\varphi(0)=\varphi^{\prime}(0)=0$ ) and its derivatives up to the second order belong to $M$, then $V^{(0)} x x(x, t)$ is continuous in $\{x \geq 0, t \geq 0\}$ and $V^{(0)}{ }_{t}$ exists is continuous in $x$, and its smoothness in $\mathrm{t}$ is determined by that of $\alpha(\mathrm{t})$ itself; (d) if in addition $\alpha \in$ $C\left(R_{+}\right)$, then $V^{(0)}(x, t) \in C^{2}(x \geq 0, t>0) \cap C(x \geq 0, t \geq 0)$ and, since the support of the distribution $\varphi \delta_{\mathrm{S} 1}$ is $S_{1}$, it follows that $\mathrm{V}^{(0)}(\mathrm{x}, \mathrm{t})$ is a classical solution of the problem (1)-(2) (with $f \equiv 0)$.

Proof is similar to that of Theorem 1 with the substitution of variables in the form: $x-\xi=2\left(\alpha_{1}(t)\right)^{1 / 2} y$.

(B) Single-layer potential $\mathrm{V}^{(1)}(\mathrm{x}, \mathrm{t})$, given by $(11)$, is a part of a solution, corresponding to the boundary values $u_{x}^{\prime}(0, t)$.

THEOREM 3. Let again condition (i) hold. Then: (a) for $\mu \in M, V(1)(x, t) \in$ M; (b) $V^{(1)}(x, t)$ is a distributional solution of the equation $L_{\alpha} u=\mu \alpha \delta_{S 2}, \quad x \geq 0, t \geq$ 0 ; satisfies zero initial condition as $t \rightarrow 0^{+}$; (c) if in addition $\alpha \in C\left(R_{+}\right)$and $\mu^{\prime} \in M$, 
then $V^{(1)}(x, t) \in C^{\infty}$ in $x$ and $C^{1}$ in $t$ for $x>0, t \geq 0$ and is a classical solution of (1) with $f=\varphi=0$; (d) $V^{(1)}(x, t)$ is continuous at $x=0$ for all $t \geq 0$.

Proof. Let us introduce a new variable in (11):

(15) $y=1 / 4 \beta_{1}(t-\tau)$.

Since $y_{\tau}^{\prime} \geq 0$ ( $=0$ only at isolated points), (15) gives an implicit function $\tau=\tau(t, y)$ with $1 /\left(4 \beta_{1}(t)\right) \leq y<+\infty$ and $\tau=0$ for $y=1 /\left(4 \beta_{1}(t)\right)$. Then, since $\alpha_{1}(t)=$ $\beta_{1}(t),(11)$ can be rewritten in the form:

$$
V^{(1)}(x, t)=\frac{1}{4 \sqrt{\pi}} \int_{1 / 4 \alpha_{1}(t)}^{\infty} \mu(\tau(t, y)) y^{-3 / 2} e^{-x^{2} y} d y
$$

(a) immediately follows from (16) since

$$
\left|\mathrm{v}^{(1)}(\mathrm{x}, \mathrm{t})\right| \leq \frac{1}{\sqrt{\pi}}\|\mu\|\left(\alpha_{1}(\mathrm{t})\right)^{1 / 2} ; \quad\left(\left(\alpha_{1}(0)=0\right) .\right.
$$

Part (b) can be proved in the way similar to that of Theorem 1, and since

$$
\left(V^{(1)}(x, t)\right)_{t}^{\prime}=1 / 2 \pi^{-1 / 2} \mu(0)\left(\alpha_{1}(t)\right)^{-1 / 2} \alpha(t) \exp \left(-x^{2} / 4 \alpha_{1}(t)\right)+V^{(1)}\left(x, t ; \mu_{t}^{\prime}\right)
$$

(where $V^{(1)}\left(x, t ; \mu_{t}^{\prime}\right)$ is the potential $(16)$ with density $\left.[\mu(\tau(t, y))]_{t}^{\prime}\right)$, part $(c)$ of this theorem is an immediate consequence of (16) and (17). For $x>0 \quad V^{(1)}(x, t)$ satisfies equation $L_{\alpha} V^{(1)}=0$ since the support of the distribution $\mu \alpha \delta_{S 2}$ is $S_{2}$, i.e. $\mu \alpha \delta_{S 2}$ is equal to 0 for $x \notin S_{2}$.

Statement (d) is obtained by comparison of the convergent integral

$$
V^{(1)}(0, t)=\frac{1}{4 \sqrt{\pi}} \int_{1 / 4 \alpha_{1}(t)}^{\infty} \mu(\cdot) y^{-3 / 2} d y
$$


with $V^{(1)}(x, t)$, given by (16), for $x$ close to 0 . This, and formulae $3.383(3), 8.359(3)$ from [3], leads to the estimate:

$$
\left|\mathrm{V}^{(1)}(\mathrm{x}, \mathrm{t})-\mathrm{V}^{(1)}(0, \mathrm{t})\right| \leq \frac{1}{2}\|\mu\||\mathrm{x}|\left(1-\Phi\left(|\mathrm{x} *| / 2\left(\alpha_{1}(\mathrm{t})\right)^{1 / 2}\right)\right),
$$

where $0 \leq x^{*} \leq x$ and $\Phi$ is the probability integral.

\section{DOUBLE-LAYER POTENTIAL}

Double-layer potential $\mathrm{W}(\mathrm{x}, \mathrm{t})$, given by (12), is a part of a solution corresponding to the boundary condition (3).

THEOREM 4. Let $\alpha$ satisfy condition (i). Then: (a) for $r \in M, W(x, t) \in$ $\mathrm{M}$; (b) $\mathrm{W}(\mathrm{x}, \mathrm{t})$ is a distributional solution of the equation $\mathrm{L}_{\alpha} \mathrm{u}=-\left(\alpha \mathrm{r} \delta_{\mathrm{S} 2}\right)_{\mathrm{x}}^{\prime}$ and satisfies zero initial condition as $t \rightarrow 0^{+}$; (c) for $x>0, t \geq 0$ if $\alpha, r \in C\left(R_{+}\right)$and $r^{\prime} \in M$, then $W(x, t) \in C^{\infty}$ in $x$ and $C^{1}$ in $t$, and it is a classical solution of (1)-(2) with $f=\varphi=0$; (d) given that $r(t) \in C^{1}\left(R_{+}\right)$W satisfies the following "jump formulae":

(18) $\lim _{x \rightarrow \pm 0} W(x, t)= \pm \frac{1}{2} r(t)$.

Proof. Parts (a)-(c) of this theorem are proved in the same way as those in Theorem 3. We introduce a new variable (15) and express $W$ in the form:

(19) $W(x, t)=\frac{x}{2 \sqrt{\pi}} \int_{1 / 4 \alpha_{1}(t)}^{\infty} r(\tau) y^{-1 / 2} e^{-x^{2} y} d y$, (where $\tau=\tau(t, y)$, as in Theorem 3 ) and its time-derivative: 
(20) $\frac{\partial \mathrm{W}}{\partial \mathrm{t}}=\frac{\mathrm{x}}{\sqrt{\pi}} \mathrm{r}(0)\left(\alpha_{1}(\mathrm{t})\right)^{-3 / 2} \alpha(\mathrm{t}) \exp \left(-\mathrm{x}^{2} / 4\left(\alpha_{1}(\mathrm{t})\right)+\mathrm{W}\left(\mathrm{x}, \mathrm{t} ; \mathrm{r}_{\mathrm{t}}\right)\right.$,

where $W\left(x, t ; r_{t}^{\prime}\right)$ is the potential (19) with density $[r(\tau(t, y))]_{t}^{\prime}$. Now part (b) can be proved applying the same technique as in Theorem 2, and (a), (c) follow from (19)-(20) as in Theorem 3.

Let us consider part (d) in more detail. First we let $r(\tau) \equiv r(t)$ for all $0 \leq \tau \leq t$, and denote the double layer potential in this case by $W_{0}$. Then, it follows from (19) and [3] (3.381, 8.359), that for $x \neq 0$

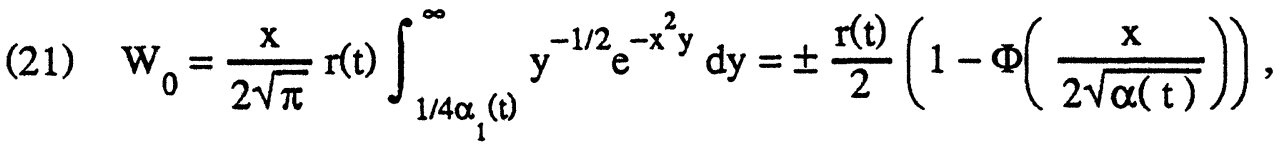

( \pm depending on the sign of $x$ ), and, since $\Phi(0)=0$,

$$
\lim _{x \rightarrow 0^{ \pm}} W_{0}(x, t)= \pm \frac{1}{2} r(t)
$$

Then, we consider the difference $\mathrm{W}_{0}-\mathrm{W}$ for $\mathrm{x}>0$, performing integration in two steps (over $(0, t-\Delta)$ and $(t-\Delta, t)$ intervals), and separately studying cases where point $t$ is "regular" (i.e., $\alpha(t)>0$ ) and "irregular" (i.e., $\alpha(t)=0$ ). Let

$$
W(x, t)-W_{0}(x, t)=I_{1}+I_{2} \text {, }
$$

where

$$
\begin{aligned}
& I_{1}=\frac{x}{4 \sqrt{\pi}} \int_{0}^{t-\Delta}(r(t)-r(\tau)) \frac{\alpha(\tau)}{\beta_{1}^{3 / 2}(t-\tau)} \exp \left(-\frac{x^{2}}{4 \beta_{1}(t-\tau)}\right) d \tau, \\
& I_{2}=\frac{x}{4 \sqrt{\pi}} \int_{t-\Delta}^{t}(r(t)-r(\tau)) \frac{\alpha(\tau)}{\beta_{1}^{3 / 2}(t-\tau)} \exp \left(-\frac{x^{2}}{4 \beta_{1}(t-\tau)}\right) d \tau,
\end{aligned}
$$

and, as in (21), for both types of $t$

$$
\left|I_{1}\right| \leq\|r\|\left[\Phi\left(\frac{x}{2 \sqrt{\alpha(t)-\alpha(t-\Delta)}}\right)-\Phi\left(\frac{x}{2 \sqrt{\alpha(t)}}\right)\right] \rightarrow 0
$$


with $\mathrm{x} \rightarrow 0$ and fixed but arbitrary $\Delta>0$.

$I_{2}$ should be estimated separately for different types of $t$. Thus, for $t$ "regular", that is $\alpha(t)>0, \Delta$ can be chosen sufficiently small so that $\alpha(\tau)>0$ over the entire interval $[t-\Delta, t]$. Then, from $\beta_{1}(t-\tau)=\alpha(\tau *)(t-\tau)$ in $[t-\Delta, t]$ and the substitution of variables $y=(t-\tau)^{-1}$, we obtain:

$$
\begin{aligned}
\left|I_{2}\right| & \leq \frac{\|\alpha\||x|\left\|r^{\prime}\right\|}{4 \sqrt{\pi} \alpha_{\Delta}^{3 / 2}} \int_{1 / \Delta}^{\infty} y^{-3 / 2} \exp \left(-\frac{x^{2}}{4(\alpha(t)-\alpha(t-\Delta))}\right) d y \\
& =\frac{\|\alpha\||x|\left\|r^{\prime}\right\|}{2 \sqrt{\pi} \alpha_{\Delta}^{3 / 2}} \sqrt{\Delta} \exp \left(-\frac{x^{2}}{4(\alpha(t)-\alpha(t-\Delta))}\right),
\end{aligned}
$$

where $0<\alpha_{\Delta}=\min _{\tau \in[t-\Delta, t]}\left|\alpha\left(\tau^{*}\right)\right| \rightarrow \alpha(t)$ with $\Delta \rightarrow 0$. As a result, $I_{2} \rightarrow 0$ with either $\mathrm{x}$ or $\Delta \rightarrow 0$. For $\mathrm{t}$ "irregular", the fact that $\alpha(\mathrm{t})=0$, requires a different approach. Using (15), we can show that

$$
\begin{aligned}
\left|I_{2}\right| & \leq \frac{1}{2} \max _{\tau \in[t-\Delta, t]}|r(t)-r(\tau)|\left(1-\Phi\left(\frac{x}{2 \sqrt{\alpha(t)-\alpha(t-\Delta)}}\right)\right) \\
& \leq \frac{1}{2} \max _{\tau \in[t-\Delta, t]}|r(t)-r(\tau)|<\varepsilon
\end{aligned}
$$

for arbitrarily small $\varepsilon>0$. These estimates imply that $\mathrm{W}_{0}-\mathrm{W} \rightarrow 0$ with $\mathrm{x} \rightarrow 0$, hence the formula (18).

\section{EXAMPLES}

(a) Let's consider the problem (1)-(3) and $\alpha(t)$ satisfying (i). Then we introduce odd extension of all functions into the region $x<0$. Then since the jumps at $x=0$ are 
$[u]_{x=0}=-2 r(t)$ and $\left[u_{x}^{\prime}\right]_{x=0}=0$, from (8) we obtain the integral representation for the solution of initial-boundary value problem (1)-(3) for $x \geq 0, t \geq 0$ :

$$
\begin{aligned}
& u(x, t)=\int_{0}^{t} d \tau \int_{0}^{\infty} f(\xi, \tau)\left(E_{\beta}(x-\xi, t-\tau)-E_{\beta}(x+\xi, t-\tau)\right) d \xi \\
& +\int_{0}^{\infty} \varphi(\xi)\left(E_{\beta}(x-\xi, t)-E_{\beta}(x+\xi, t)\right) d \xi+\left.2 \int_{0}^{t} \alpha(\tau) r(\tau) \frac{\partial}{\partial \xi}\left(E_{\beta}(-\xi, t-\tau)\right)\right|_{\xi=0} d \tau .
\end{aligned}
$$

Function $u(x, t)$ satisfies the equation (1) and initial and boundary conditions (2)-(3), given that the functions $\alpha, \mathrm{r}, \varphi, \mathrm{f}$ satisfy restrictions discussed in Theorems 1-4.

(b) As in a), considering the problem (1)-(3) for $0<x<b$ with additional condition $u(b, t)=h(t)$, we find solution $u(x, t)$ in the form (with $\alpha(t)$ still satisfying (i)):

$$
u(x, t)=V(x, t)+V^{(0)}(x, t)+W_{1}(x, t)+W_{2}(x, t)
$$

where double-layer potentials $\mathrm{W}_{1}$ (the same as in (12)) and $\mathrm{W}_{2}$ have density functions 2 $r(t)$ and $\mu(t)$ respectively. $W_{2}$ is concentrated on the $x=1$ part of the boundary and is given by the formula:

$$
W_{2}(x, t)=\int_{0}^{t} \alpha(\tau) \mu(\tau) \frac{\partial}{\partial \xi}\left[E_{\beta}(x-\xi, t-\tau)-E_{\beta}(x+\xi, t-\tau)\right]_{\xi=b} d \tau
$$

Using (18) for $\mathrm{W}_{1}$ we find that $\mathrm{u}$ (22) satisfies the conditions (2)-(3) (note that $\left.\mathrm{W}_{2}(0, t)=0\right)$. Applying then the boundary condition $u(b, t)=h(t)$ to $(22)$ and using the "jump formula" for $\mathrm{W}_{2}$ we obtain:

$$
h(t)=V(b, t)+V^{(0)}(b, T)+W_{1}(b, t)-\frac{1}{2} \mu(t)
$$




$$
+\frac{1}{2 \sqrt{\pi}} \int_{0}^{t} \alpha(\tau) \mu(\tau)\left(\beta_{1}(t-\tau)\right)^{-3 / 2} \exp \left(-b^{2} / 4 \beta_{1}(t-\tau)\right) d \tau .
$$

The density $\mu(t)$ has to be found from the linear Volterra integral equation of the second kind:

$$
\mu(t)=\int_{0}^{t} k(t, \tau) \mu(\tau) d \tau+F(t) \equiv K[\mu]
$$

with continuous $F(t)$ (Theorems 1-4) and a kernel

$$
k(t, \tau)=\frac{1}{\sqrt{\pi}} \alpha(\tau)\left(\beta_{1}(t-\tau)\right)^{-3 / 2} \exp \left(-b^{2} / 4 \beta_{1}(t-\tau)\right) .
$$

The unique solvability of the equation (23) can be obtained by methods discussed in [3], or it can be proved that some power $\mathrm{K}^{\mathrm{m}}$ of the operator $\mathrm{K}$ is a contraction on $\mathrm{C}[0, \mathrm{~T}]$. So, equation (23) has a unique solution, which can be found by the method of successive approximations, and formula (22) gives its integral representation .

(c) Considering (1)-(2) with $\alpha(t)$ satisfying (ii), $f=0$ and $\varphi$ being an odd extension into $\mathrm{x}<0$, we can find the solution in the form

$$
\begin{aligned}
u(x, t) & =E * \bar{\varphi} \delta_{S_{1}}=\int_{0}^{\infty} \varphi(\xi)\left(E_{\beta}(x-\xi, t)-E_{\beta}(x+\xi, t)\right) d \xi \\
& =\int_{0}^{\infty} \frac{\varphi(\xi)}{2 \sqrt{\pi \alpha_{1}(t)}}\left[\exp \left(-\frac{(x-\xi)^{2}}{4 \alpha_{1}(t)}\right)-\exp \left(-\frac{(x+\xi)^{2}}{4 \alpha_{1}(t)}\right)\right] d \xi
\end{aligned}
$$

Verification is straightforward. As an example of $\alpha(t)$ satisfying (ii) $1 / 2+\cos (t)$ may do. Under the condition (ii) equation (1), not being of parabolic type, still can be solved in the form of a convolution of its fundamental solution with a single layer (Theorem 2).

\section{REFERENCES}

[1] I. Malyshev, On the inverse problem for a heat-like equation, J. of Appl. Math. and Simulation, Vol. 1, No. 2: 81-97, (1987). 
[2] V.S. Vladimirov, Equations of Mathematical Physics, Marcel Dekker, (1971).

[3] I.S. Gradshteyn, I.M. Ryzhik, Table of Integrals, Series and Products, Academic Press, (1980).

[4] I. Malyshev, Description of the parabolic potentials in the non-cylindrical domains by the Sobolev-Schwartz's Distributions, J. of Comput. and Appl.Math., 30: 58-66, (1976). [Russian; English translation: MR 58, \# 1196]. 


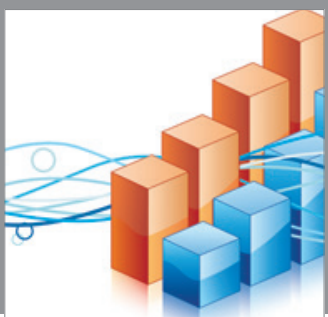

Advances in

Operations Research

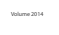

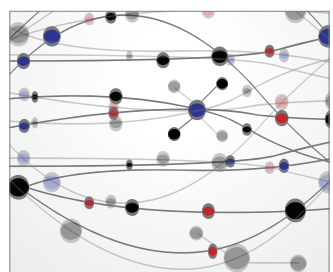

\section{The Scientific} World Journal
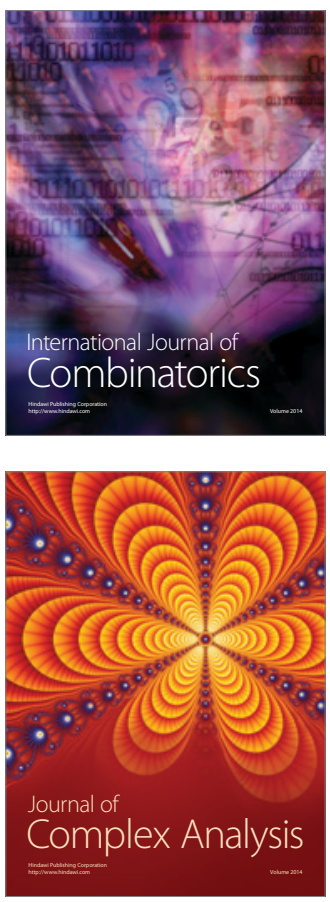

International Journal of

Mathematics and

Mathematical

Sciences
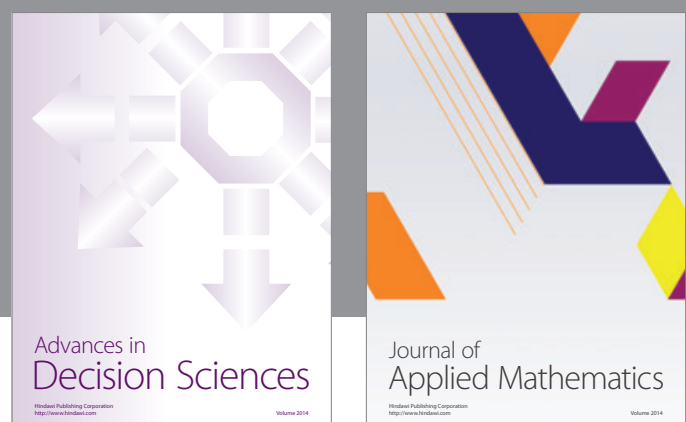

Journal of

Applied Mathematics
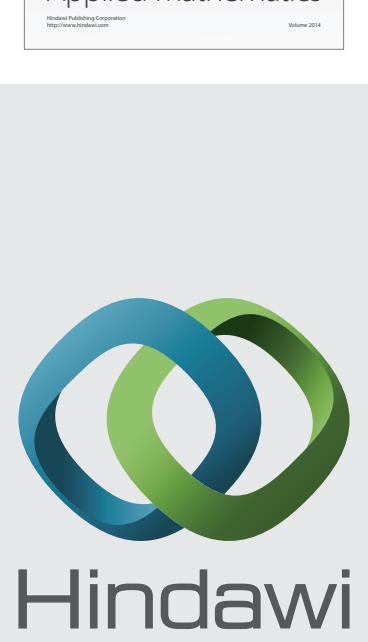

Submit your manuscripts at http://www.hindawi.com
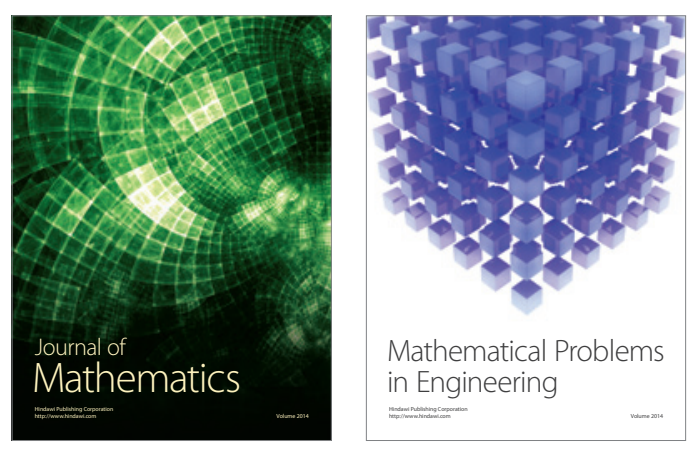

Mathematical Problems in Engineering
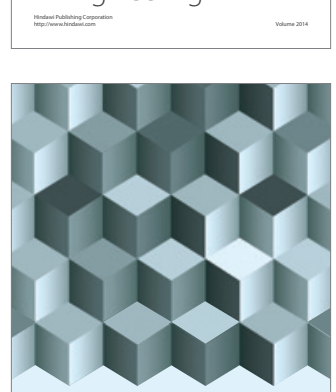

Journal of

Function Spaces
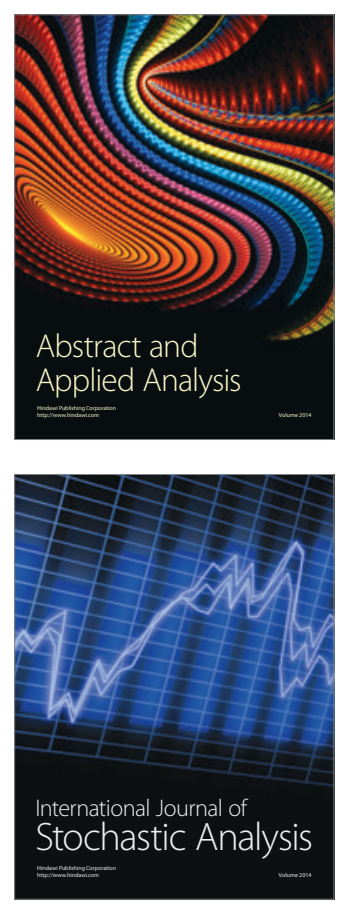

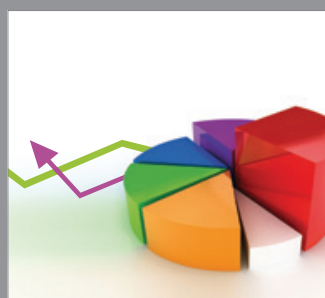

ournal of

Probability and Statistics

Promensencen
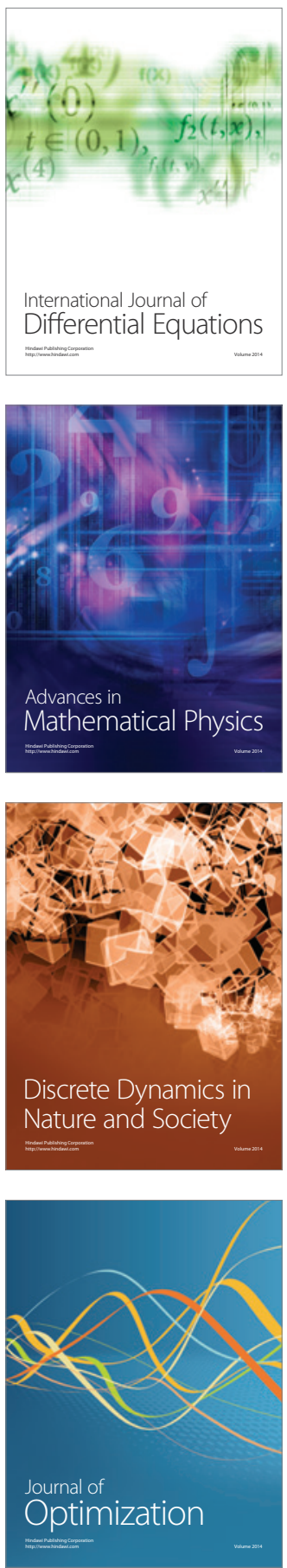